\title{
Organ roles for mitochondrial mutations?
}

the mutation
loci are
focused
around the
origin of
replication
of mtDNA

Variation in mitochondrial genomes is known to occur between individuals through maternal inheritance of distinct mitochondrial DNA (mtDNA) and within individuals through somatic mtDNA mutations. Although most studies have investigated roles for mtDNA variation in ageing and disease, a new study now suggests that somatic mtDNA mutations occur recurrently in particular human organs and thus may have specific roles in normal tissue physiology.

To investigate the degree and the distribution of intra-individual variation in mtDNA (which is known as heteroplasmy), Samuels et al. carried out high-throughput sequencing of mtDNA from ten normal human tissue types in each of two individuals. They also analysed similar published data sets from an additional two individuals. Interestingly, they found that somatic mtDNA mutations were distributed nonrandomly among tissues. For some tissues (such as the lung and large intestine) no somatic mtDNA mutations were identified, whereas other tissues (such as the liver, kidney and skeletal muscle) harboured particular somatic mtDNA mutations in up to $13 \%$ of mtDNA copies.
The identified mutations were primarily single-nucleotide variants (SNVs) and were surprisingly recurrent: nine SNVs were found in the same tissue in more than one individual, and the liver and kidney shared almost identical repertoires of SNVs, despite these organs being derived from distinct embryonic tissue types. These recurrent patterns show that the same somatic mtDNA mutations are occurring independently in multiple tissues and individuals.

What are the biological implications of the observed mutation patterns? The authors showed that the mutation loci are focused around the origin of replication of mtDNA and that the tissues with these recurrent mutations (that is, the liver, kidney and skeletal muscle) have the greatest numbers of mitochondria per cell among the tissues studied. Thus, the authors hypothesize that these somatic mutations have a regulatory effect on mtDNA replication and are positively selected in tissues that require high numbers of mitochondria for physiological functions.

It will be interesting to test this intriguing hypothesis - and to rule out the idea that these mutations are merely passive consequences of mtDNA replication or of mutagenesis in these tissues - by dissecting the functional consequences of these mtDNA mutations in model systems. Furthermore, as the liver, kidney and skeletal muscle are commonly associated with mitochondrial diseases, potential pathological consequences of these somatic mtDNA mutations will be key to investigate.

Darren J. Burgess

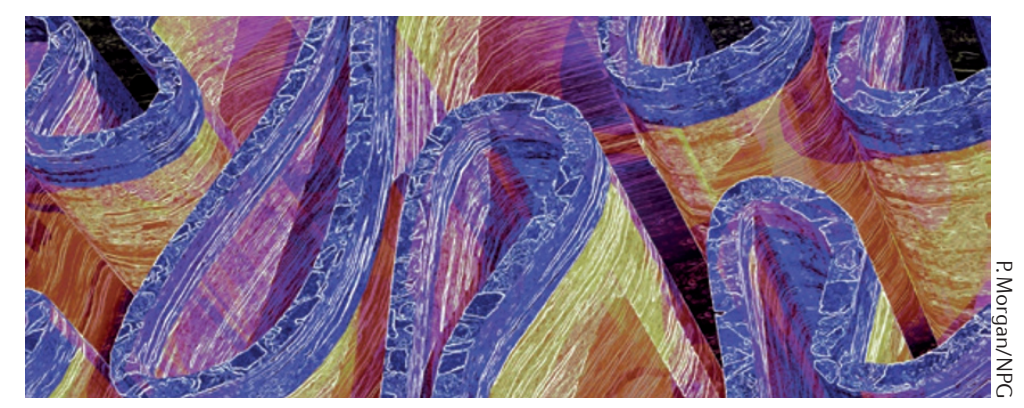

ORIGINAL RESEARCH PAPER Samuels, D. C.

et al. Recurrent tissue-specific mtDNA mutations are common in humans. PLoS Genet. 9, e1003929 (2013)

FURTHER READING Schon, E. A., DiMauro, S. \& Hirano, M. Human mitochondrial DNA: roles of inherited and somatic mutations. Nature Rev. Genet. 13, 878-890 (2012) 